PROCEEDINGS OF THE

AMERICAN MATHEMATICAL SOCIETY

Volume 129, Number 6, Pages 1825-1831

S 0002-9939(00)05766-X

Article electronically published on October 31, 2000

\title{
THE POSTNIKOV TOWER AND THE STEENROD PROBLEM
}

\author{
MING-LI CHEN
}

(Communicated by Ralph Cohen)

\begin{abstract}
The Steenrod problem asks: given a $G$-module, when does there exist a Moore space realizing the module? By using the equivariant Postnikov Tower, it is shown that a $\mathbb{Z} G$-module is $\mathbb{Z} G$-realizable if and only if it is $\mathbb{Z H}$ realizable for all $p$-Sylow subgroups $H$, for all primes $p \| G \mid$.
\end{abstract}

Let $G$ be a finite group. Let $M$ be a finitely generated $\mathbb{Z} G$-module. We say that $M$ is a Steenrod representation if there exists a Moore space $X$ with $G$-action such that the homology of $X$ is isomorphic to $M$ as a $\mathbb{Z} G$-module. Recall that a Moore space is a topological space whose reduced homology vanishes in all dimensions except one. Without loss of generality, $M$ will be assumed $\mathbb{Z}$-free [1] from now on. In this paper, the following statement will be proved:

$M$ is a Steenrod representation as a $\mathbb{Z} G$-module if and only if $M$ is a

Steenrod representation as a $\mathbb{Z} H$-module for all p-Sylow subgroups $H$ of

$G$, for all primes $p \| G \mid$.

This statement is inspired by papers by J. Arnold 1, 2] and P. Vogel 18. Arnold 2] showed that if $G$ is cyclic, then every finitely generated $\mathbb{Z} G$-module is a Steenrod representation. And Vogel showed that if for any $\mathbb{Z} G$-module $M$, we can always find a $G$-Moore space $X$ realizes $M$, then $G$ has only cyclic Sylow subgroups.

Let us first consider a Moore space $X$ whose homology is isomorphic to $M$ as a $\mathbb{Z}$-module. Let $G(X)$ denote the space of self homotopy equivalences of $X$ and let $B_{G(X)}$ [9] denote the classifying space of the $H$-space $G(X)$ for a certain fibration defined by Dold and Lashof [10]; see also Stasheff [15]. Since a homotopy equivalence of $X$ induces an automorphism on $M$, there is a map $G(X) \rightarrow A u t(M)$. And the map $G(X) \rightarrow \operatorname{Aut}(M)$ induces another map $\sigma: B_{G(X)} \rightarrow B_{A u t(M)}$. On the other hand, since $M$ is a $\mathbb{Z} G$-module, there is a map $G \rightarrow A u t(M)(=G L(n, \mathbb{Z})$ if $M=\mathbb{Z}^{n}$ ), which induces $\varphi: B_{G} \rightarrow B_{A u t(M)}$. Following Cooke's theory [9], as pointed out in 12, $M$ is a Steenrod representation as a $\mathbb{Z} G$-module if and only if the lifting $\varphi_{\infty}$ exists in

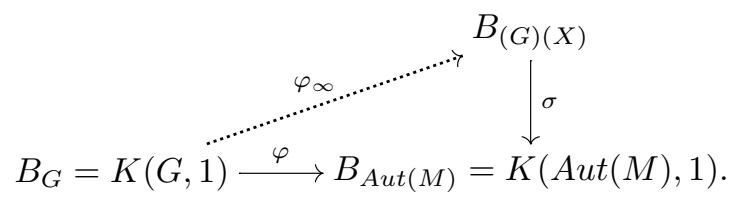

Received by the editors June 18, 1997 and, in revised form, September 13, 1999.

1991 Mathematics Subject Classification. Primary 55R91, 55S45, 55S91.

(C)2000 American Mathematical Society 
We will follow this path to show the lifting exists in (1) provided the lifting exists for $B_{H}$ for all $p$-Sylow subgroups $H$ of $G$, for all primes $p \| G \mid$ :

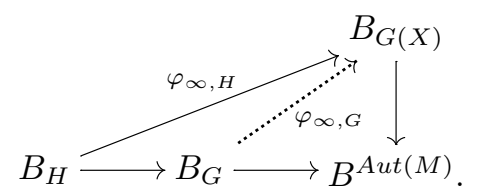

Note that we do not assume the same space $X$ is chosen for different Sylow subgroups of $G$. In fact, we work on $B_{G(X)}$, the classifying space of the $H$-space $G(X)$ (references: 10] Dold and Lashof and [15] Stasheff). $B_{G(X)}$ is homeomorphic to $B_{G(Y)}$ if $X$ is homotopy equivalent to $Y$. Following Cooke [9] (see also Kahn [12]), if the lifting

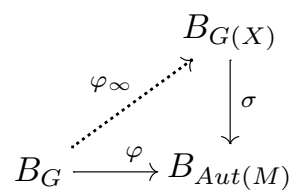

exists, then there is a space $Y$ homotopy equivalent to $X$ such that $G$ acts on $Y$, and $Y$ realizes $M$. The idea to show the lifting exists in (1) is to use the Postnikov Tower to build the lifting step by step. But the usual Postnikov Tower does not apply because in the first level $\sigma: B_{G(X)} \rightarrow B_{A u t(M)}$ of the tower the base space $B_{A u t(M)}$ has a non-trivial fundamental group $\pi_{1}\left(B_{A u t(M)}\right)=A u t(M)$. We modify this by working on the universal covering spaces and in the equivariant category instead. Let $E_{G}$ denote the universal covering space of $B_{G}$. And let $E_{G(X)}$ denote the universal covering space of $B_{G(X)}$. In fact, $E_{G(X)}$ is the pullback $\sigma^{*} E_{A u t(M)}$ of $\sigma: B_{G(X)} \rightarrow B_{A u t(M)}$. Then there are induced maps on covering spaces $\widetilde{\varphi}: E_{G} \rightarrow E_{A u t(M)}$ and $\widetilde{\sigma}: E_{G(X)} \rightarrow E_{A u t(M)}$. We will construct an equivariant lifting $\widetilde{\varphi}_{\infty}: E_{G} \rightarrow E_{G(X)}$ in the following diagram:

$$
\begin{array}{cc} 
& E_{G(X)} \\
E_{G} \stackrel{\neg}{ } \stackrel{\tau}{ } \stackrel{\tilde{\varphi}}{\longrightarrow} E_{\text {Aut }(M)} .
\end{array}
$$

And that is sufficient for the proof of the main statement: by taking the quotient of (21) by suitable group actions,

$$
\begin{aligned}
& \begin{array}{ccccc} 
& & E_{G(X)} / G & \rightarrow & E_{G(X)} / \operatorname{Aut}(M) \\
E_{G} / G & \nearrow & \downarrow & & \\
\downarrow & E_{\text {Aut }(M)} / G & \rightarrow & E_{\text {Aut }(M)} / \operatorname{Aut}(M)
\end{array} \Rightarrow \\
& E_{G(X)} / \operatorname{Aut}(M)=B_{G(X)} \\
& B_{G}=E_{G} / G \stackrel{\nearrow}{\longrightarrow} E_{\text {Aut }(M)} / \operatorname{Aut}(M)=B_{\text {Aut }(M)}
\end{aligned}
$$

we obtain (11).

In Postnikov theory, one of the key ingredients is the identification of $H^{n}(X ; \pi)$ with $[X ; K(\pi, n)]$. There is a counterpart in the equivariant setting: In [11], Theorem 24.1, Eilenberg showed that the cohomology (a) $H^{n}(X ; \pi)$ defined by using 
local coefficient (i.e. $\pi_{1}(X)$ acting on $\pi$ ) is the same as (b) $H^{n}(Y ; \pi)$, the homology of the cochain complex $\operatorname{Hom}_{\pi_{1}(X)}\left(C_{n}(Y), \pi\right)$, with $\pi_{1}(X)$ acting on both $Y$ and $\pi$, where $Y$ is the universal covering space of $X$. Bredon [5] then showed that $H^{n}(Y ; \pi)$ can be identified with (c) $[Y ; K(\pi, n)]_{G}$, the equivariant homotopy classes of equivariant maps; see also [6] p. 268. Note that the $G$-action on $K(\pi, n)$ has a fixed point. In summary, using the above notation, we have

Proposition. The following cohomology groups are isomorphic:

(a) $H^{n}(X ; \pi)$,

(b) $H^{n}(Y ; \pi)$ and,

(c) $[Y ; K(\pi, n)]_{G}$.

For example, the group cohomology $H^{n}(G ; \pi)=H^{n}\left(B_{G} ; \pi\right)$ is equal to $\left[E_{G}\right.$; $K(\pi, n)]_{G}$. We will use the three equivalent cohomology groups (a), (b) and (c) interchangeably depending on the circumstances.

The arguments in the usual Postnikov theory will carry through in the equivariant setting. This is explained in the following: Let $X$ be a topological space and $\pi_{1}(X) \neq 0$. Let $\pi_{n}$ denote $\pi_{n}(X)$. Consider the fibration $F_{1} \rightarrow X \rightarrow B_{\pi_{1}}$ with $\pi_{1}$ acting on the fibre $F_{1}$ and consider the spectral sequence associated with this fibration. The fundamental class $\iota_{2}$ of $H^{2}\left(F_{1} ; \pi_{2}\right)$ [14 is invariant under $\pi_{1}$, so it is an element in $H^{0}\left(\pi_{1} ; H^{2}\left(F_{1} ; \pi_{2}\right)\right) . \iota_{2}$, considered as an element in $E_{3}^{0,2}$, transgresses to $\tau\left(\iota_{2}\right) \in H^{3}\left(\pi_{1} ; \pi_{2}\right)=E_{3}^{3,0}$. We can regard $\tau\left(\iota_{2}\right) \in H^{3}\left(\pi_{1} ; \pi_{2}\right)$ as an element in $\left[E_{\pi_{1}} ; K\left(\pi_{2}, 3\right)\right]_{\pi_{1}}$, i.e. a $\pi_{1}$-equivariant map $\tau\left(\iota_{2}\right): E_{\pi_{1}} \rightarrow K\left(\pi_{2}, 3\right)$. Now we switch to covering spaces and the equivariant setting. As in the simply-connected case of Postnikov theory, the composition $E_{X} \rightarrow E_{\pi_{1}} \stackrel{\tau\left(\iota_{2}\right)}{\longrightarrow} K\left(\pi_{2}, 3\right)$ is equivariantly trivial, for it corresponds to the edge homomorphism

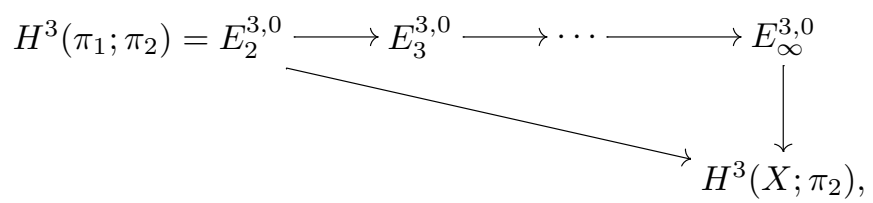

which maps $\tau\left(\iota_{2}\right)$ to zero in $H^{3}\left(X ; \pi_{2}\right)$. So the lifting $\widetilde{\varphi}_{2}: E_{X} \rightarrow E_{2}$ exists in

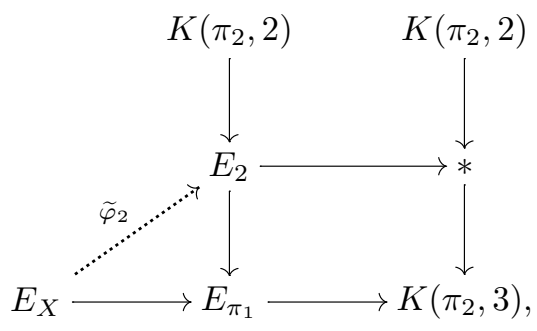

where $K\left(\pi_{2}, 2\right) \rightarrow E_{2} \rightarrow E_{\pi_{1}}$ is the pullback of $K\left(\pi_{2}, 2\right) \rightarrow * \rightarrow K\left(\pi_{2}, 3\right)$ and $*$ denotes an equivariantly contractible space.

It can be shown, as in the simply-connected case of Postnikov theory, that $\widetilde{\varphi}_{2}$ induces isomorphisms on homotopy groups for dimension $\leq 2$, and zero otherwise. Now, take the quotient of $\widetilde{\varphi}_{2}: E_{X} \rightarrow E_{2}$ by $\pi_{1}$-action, we get $\varphi_{2}: X \rightarrow E_{2} / \pi_{1}$. Then consider the fibration $F_{2} \rightarrow X \rightarrow E_{2} / \pi_{1}$. Now we are back to the situation where we started with the fibration $F_{1} \rightarrow X \rightarrow B_{\pi_{1}}$. The argument can be repeated. 
Summing up, we have a sequence of equivariant maps and free spaces

$$
E_{X} \rightarrow \cdots \rightarrow E_{n} \rightarrow \cdots \rightarrow E_{2} \rightarrow E_{1}=E_{\pi_{1}}
$$

and when we take the quotient of the sequence by $\pi_{1}$ action, we get the Postnikov Tower of $X$

$$
X \rightarrow \cdots \rightarrow E_{n} / \pi_{1} \rightarrow \cdots \rightarrow E_{2} / \pi_{1} \rightarrow E_{1} / \pi_{1}=B_{\pi_{1}}
$$

in the non-simply-connected case. Note

$$
\pi_{i}\left(E_{n}\right)=\left\{\begin{array}{cc}
\pi_{i}\left(E_{X}\right)=\pi_{i}(X) & 1<i \leq n \\
0 & \text { otherwise }
\end{array}\right.
$$

For the proof of the main theorem, we start with

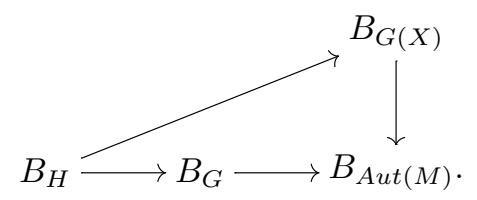

The corresponding universal covering spaces and equivariant maps are:

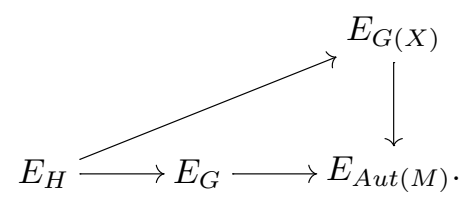

Consider the spectral sequence associated with the fibration $F_{1} \rightarrow B_{G(X)} \rightarrow$ $B_{A u t(M)}$. Let $\pi_{i}$ denote $\pi_{i}\left(B_{G(X)}\right)$. Since $\pi_{1}\left(B_{G(X)}\right)=\pi_{0}(G(X))=\operatorname{Aut}(M)=$ $\pi_{1}\left(B_{\text {Aut }(M)}\right)$ (see [12, Theorem 1.1(a)),

$$
\pi_{i}\left(F_{1}\right)=\left\{\begin{array}{cc}
0, & i=1 \\
\pi_{i}, & i \geq 2
\end{array}\right.
$$

Let $\iota_{2}$ be the fundamental class of $H^{2}\left(F_{1}, \pi_{2}\right) . \iota_{2}$ is invariant under the action of Aut $(M)$, i.e. $\iota_{2}$ is an element in $H^{0}\left(B_{A u t(M)} ; H^{2}\left(F_{1} ; \pi_{2}\right)\right)$. Then the transgression $\tau\left(\iota_{2}\right)$ is an element in $H^{3}\left(B_{A u t(M)}, \pi_{2}\right)$, which can be regarded as an element in $\left[E_{A u t(M)} ; K\left(\pi_{2}, 3\right)\right]_{A u t(M)}$. Since we assume $M$ is a Steenrod representation as a $\mathbb{Z} H$-module, the lifting $\varphi_{H, \infty}$ exists.

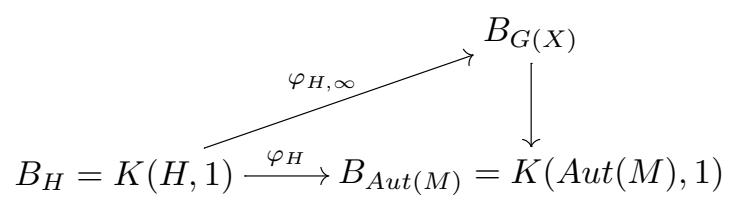


Hence there exists a sequence of liftings $\left\{\widetilde{\varphi}_{n, H}\right\}$.

$$
\begin{array}{ccccc} 
& E_{G(X)} & & \\
E_{H} \stackrel{\widetilde{\varphi}_{\infty, H}}{\stackrel{\widetilde{\varphi}_{n, H}}{\longrightarrow}} & \vdots & & \\
& E_{n} & \stackrel{\tau\left(\iota_{n+1}\right)}{\longrightarrow} & K & \\
\widetilde{\varphi}_{n-1, H} & \downarrow & & \\
& E_{n-1} & \stackrel{\tau\left(\iota_{n}\right)}{\longrightarrow} & K & K\left(\pi_{n}, n+1\right) \\
& \downarrow & & \\
\vdots & & \\
& E_{\text {Aut }(M) .} .
\end{array}
$$

The transgression $\tau\left(\iota_{2}\right)$ is then zero in the pullback $F_{1} \rightarrow \varphi_{H}^{*}\left(B_{G(X)}\right) \rightarrow B_{H}$ of $\varphi_{H}: B_{H} \rightarrow B_{A u t(M)}$. Hence the composition $E_{H} \rightarrow E_{G} \rightarrow E_{A u t(M)} \rightarrow K\left(\pi_{2}, 3\right)$ is equivariantly homotopy equivalent to a trivial map. In other words, in the sequence of maps $\left[E_{\text {Aut }(M)} ; K\left(\pi_{2}, 3\right)\right]_{A u t(M)} \rightarrow\left[E_{G} ; K\left(\pi_{2}, 3\right)\right]_{G} \rightarrow\left[E_{H} ; K\left(\pi_{2}, 3\right)\right]_{H}, \tau\left(\iota_{2}\right)$ goes to zero, i.e. the element $\tau\left(\iota_{2}\right) \circ \widetilde{\varphi}_{G}$ restricts to zero in the map $\left[E_{G} ; K\left(\pi_{2}, 3\right)\right]_{G} \rightarrow$ $\left[E_{H} ; K\left(\pi_{2}, 3\right)\right]_{H}$, which is identified with $H^{3}\left(G ; \pi_{2}\right) \rightarrow H^{3}\left(H ; \pi_{2}\right)$. Since $\tau\left(\iota_{2}\right) \circ \widetilde{\varphi}_{G}$ restricts to zero via the restriction maps $H^{3}\left(G ; \pi_{2}\right) \rightarrow H^{3}\left(H ; \pi_{2}\right)$ for all $p$-Sylow subgroups $H, \tau\left(\iota_{2}\right) \circ \widetilde{\varphi}_{G}$ is a zero element in $H^{3}\left(G ; \pi_{2}\right)$. So the pull-back $\widetilde{\varphi}_{G}^{*}\left(E_{2}\right)$ of the map $\tau\left(\iota_{2}\right) \circ \widetilde{\varphi}_{G}$ is a trivial bundle. Therefore the lifting $\widetilde{\varphi}_{2, G}$ exists. Note that the liftings are in one-to-one correspondence with elements in $\left[E_{G} ; K\left(\pi_{2}, 2\right)\right]_{G}$. Let $\theta_{2, G}$ denote the element in $\left[E_{G} ; K\left(\pi_{2}, 2\right)\right]_{G}$ corresponding to $\widetilde{\varphi}_{2, G}$, as illustrated in the following diagram:

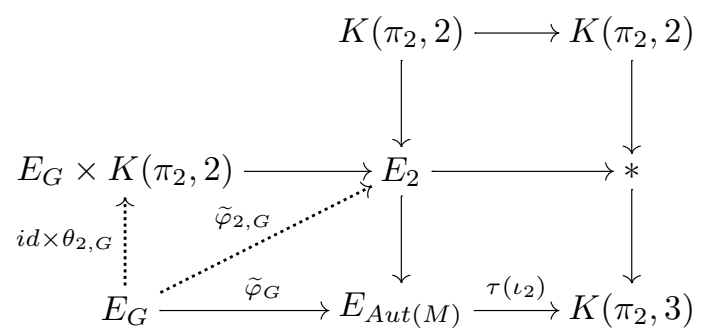

The above diagram is in the equivariant category, where $E_{2}$ is the second level of the Postnikov Tower of $E_{G(X)}$.

Now the problem is to choose a right lifting $\widetilde{\varphi}_{2, G}$ (or $\theta_{2, G}$ ) so that the diagram

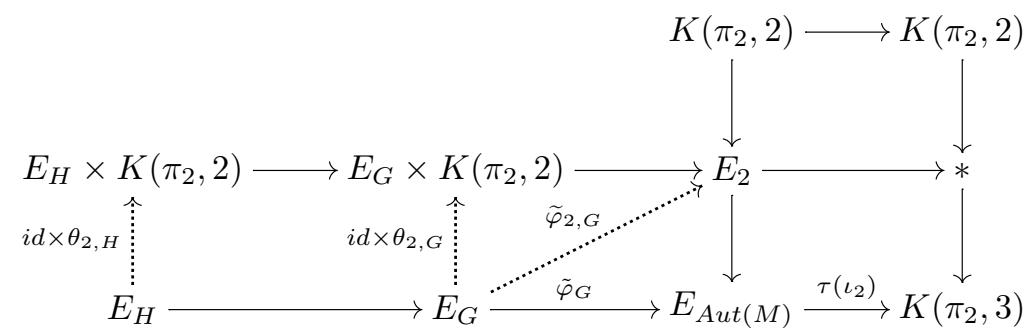

commutes for all $p$-Sylow subgroups $H$. If the above diagram commutes, then we can repeat the previous argument: $\left[\tau\left(\iota_{3}\right) \circ \widetilde{\varphi}_{2, G}\right] \in H^{4}\left(G ; \pi_{3}\right)$ restricts to $\left[\tau\left(\iota_{3}\right) \circ\right.$ $\left.\widetilde{\varphi}_{2, H}\right]=0$ in $H^{4}\left(H ; \pi_{3}\right)$ for all $p$-Sylow subgroups $H$. So $\left[\tau\left(\iota_{3}\right) \circ \widetilde{\varphi}_{2, G}\right]=0$ in $H^{4}\left(G ; \pi_{3}\right)=\left[E_{G} ; K\left(\pi_{3}, 4\right)\right]_{G}$ and then we can go inductively. But the desired lift 
$\theta_{2, G}$ may not exist, unless we have a right $\theta_{2, H}$, i.e. $\theta_{2, H}$ has to be in the image of the restriction map $H^{2}\left(G ; \pi_{2}\right) \rightarrow H^{2}\left(H ; \pi_{2}\right)$. Such a $\theta_{2, H}$ is said to be $G$-invariant in $H^{2}\left(H ; \pi_{2}\right)$. In the following, we will show how to alter all the liftings $\left\{\widetilde{\varphi}_{n, H}\right\}$ to ensure $\left\{\theta_{n, H}\right\}$ be $G$-invariant. We take the new $\theta_{2, H}$ to be $\frac{1}{[G: H]} \Sigma_{G=\cup g H} g \theta_{2, H} g^{-1}$, i.e. $\frac{1}{[G: H]}$ res $\circ \operatorname{tr} \theta_{2, H}$. The corresponding new lift $\widetilde{\varphi}_{2, H}$

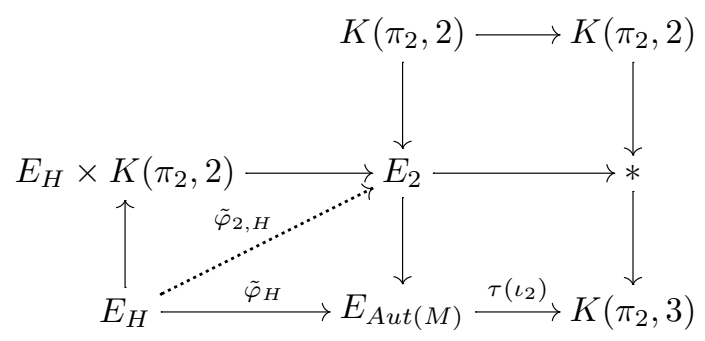

is $\left(\widetilde{\varphi}_{H}, \frac{1}{[G: H]}\right.$ resotr $\left.\theta_{2, H}\right)$. (The lifting $\widetilde{\varphi}_{2, H}$ that corresponds to $\theta_{2, H}$ is $\left(\widetilde{\varphi}_{H}, \theta_{2, H}\right)$.) Now we use the fact that $E_{G(X)}$ is an $\mathcal{H}$-space (a space with homotopy mutiplication), $E_{n}$ 's are $\mathcal{H}$-spaces and $\tau\left(\iota_{n}\right)$ 's are $\mathcal{H}$-maps (see [19]). Therefore $\tau\left(\iota_{3}\right) \circ$ $\left(\widetilde{\varphi}_{H}, \frac{1}{[G: H]}\right.$ res $\left.\circ \operatorname{tr} \theta_{2, H}\right)=\tau\left(\iota_{3}\right) \circ\left(\frac{1}{[G: H]} r e s \circ \operatorname{tr}\left(\widetilde{\varphi}_{H}, \theta_{2, H}\right)\right)=\frac{1}{[G: H]} r e s \circ \operatorname{tr} \tau\left(\iota_{3}\right) \circ$ $\left.\left(\widetilde{\varphi}_{H}, \theta_{2, H}\right)\right)=0$. In general, we will change $\widetilde{\varphi}_{n, H}=\left(\widetilde{\varphi}_{H}, \theta_{2, H}, \cdots, \theta_{n, H}\right)$ to $\left(\widetilde{\varphi}_{H}, \frac{1}{[G: H]}\right.$ resotr $\theta_{2, H}, \frac{1}{[G: H]}$ resotr $\left.\theta_{n, H}\right)$. Then the new $\theta_{n, H}$, i.e. $\frac{1}{[G: H]}$ resotr $\theta_{n, H}$ will be $G$-invariant.

\section{ACKNOWLEDGEMENTS}

The auther would like to thank Professor Amir Assadi for his encouragement and advice, Jeff Strom for many helpful and informative conversations and the referee for suggestions on clarifying a few points.

\section{REFERENCES}

1. J. Arnold, A Solution of a Problem of Steenrod for Cyclic Groups of Prime Order, Proc. Amer. Math. Soc. 62 (1977), 177-182. MR 55:4152

2. J. Arnold, On Steenrod's Problem for Cyclic p-Groups, Canad. J. Math. 29 (1977), 421-428. MR 55:13411

3. A. Assadi, Homotopy Actions and Cohomology of Finite Groups, Transformation Groups, Poznań 1985, Springer, LNM 1217. MR 88d:55005

4. D. Benson and N. Habegger, Varieties for Modules and a Problem of Steenrod, J. Pure and Applied Algebra, 44 (1987), 13-34. MR 88e:55020

5. G. Bredon, Equivariant Cohomology Theories, LNM 34, 1967. MR 35:4914

6. G. Bredon, Equivariant Cohomology Theories, Bull. AMS 73 (1967), 266-268. MR 34:6762

7. K. Brown, Cohomology of Groups, Springer-Verlag, 1982. MR 83k:20002

8. G. Carlsson, A Counterexample to a Conjecture of Steenrod, Invent. Math. 64 (1981) 171-174. MR 82j:57036

9. G. Cooke, Replacing Homotopy Actions by Topological Actions, Trans. AMS 237 (1978), 391-406. MR 57:1529

10. A. Dold and R. Lashof, Principal Qusi-fibrations and Fibre Homotopy Equivalence of Bundles, Illinois J. Math. 3 (1959), 285-305. MR 21:331

11. S. Eilenberg, Homology of Spaces with Operators I, Trans. AMS 61 (1947), 378-417; errata, 62 (1947), 548. MR 9:52b

12. P. Kahn, Steenrod Problem and $k$-invariants of Certain Classifying Spaces, Algebraic KTheory, Proc. Oberwolfach 1980, Part 2, LNM, 967. MR 84k:57030

13. R. Lashof, Problems in Differential and Algebraic Topology, Seattle Topology Conference, Ann. Math. (1965). MR 32:443 
14. R. Mosher and M. Tangora, Cohomology Operations and Applications in Homotopy Theory, Harper \& Row, 1968. MR 37:2223

15. J. Stasheff, A Classification Theorem for Fibre Spaces, Topology 2 (1963), 239-246. MR 27:4235

16. R. Swan, Invariant Rational Functions and A Problem of Steenrod, Invent. Math. 7 (1969), 148-158. MR 39:5532

17. P. Vogel, On Steenrod Problem for Non-abelian Finite Groups, Proc. Alg. Top. Conf. Aarhus 1982, Springer LNM 1051 (1984). MR 86e:55015

18. P. Vogel, A Solution of the Steenrod Problem for G-Moore Spaces, K-Theory 1 (1987), 325335. MR 89e:57033

19. G.W. Whitehead, Elements of Homotopy Theory, Springer-Verlag, 1978. MR 80b:55001

Center for the Mathematical Sciences, University of Wisconsin, Madison, Wisconsin 53715

E-mail address: mchen@cms.wisc.edu 